\title{
Foreword: Immunogenetics special issue 2017
}

\author{
Ronald E. Bontrop ${ }^{1}$
}

Published online: 10 July 2017

(C) Springer-Verlag GmbH Germany 2017

Since its inception, the scientific journal Immunogenetics has been publishing a large number of papers dedicated to the immunobiology of MHC genes and gene products. In the first decades, the focus was mainly on the characterisation of MHC gene products and their role in transplantation, both in humans and in rodent models. In later years, attention shifted towards understanding the biological role of the $\mathrm{MHC}$ genes in a variety of biological processes such as autoimmune and infectious diseases, as well as in reproductive biology. In a relatively short time, however, the methods of characterisation have changed dramatically. In the early days, serology was the technique of choice, to be bypassed by the introduction of various biochemical and molecular methods. At the present time, a massive amount of information is able to be gathered in a relatively short period by new technologies such as next-gen sequencing. One other feature has changed as well. The number of species being studied has exploded. This is an aspect of comparative genetics that has taught us a great deal about the evolution of the MHC multigene family and its ligands, and is greatly appreciated by the readers of Immunogenetics. After the discovery of the two lineages of T-cell receptors, particular MHC class I epitopes were found to be ligands for KIR receptors on NK cells. This opened a complete new avenue of research. The editors are pleased to announce that this year's special issue of Immunogenetics is devoted to the role of $\mathrm{MHC} / \mathrm{KIR}$ genes in health and disease, and

Ronald E. Bontrop

bontrop@bprc.nl

1 Biomedical Primate Research Center, PO Box 3306, 2280 Rijswijk, GH, Netherlands we are proud to present reviews provided by a selected group of authorities in the field.

The first contribution to set the stage introduces the key players as well as their main known functions (Kelly and Trowsdale 2017). As we have learned from the AIDS pandemic, MHC and KIR genes each play an important role in controlling either susceptibility or resistance to infectious disease. As regards AIDS, the current state of knowledge in the field of immuogenetics has been reviewed for humans (Carrington and Naranbhai 2017) and chimpanzees (de Groot et al. 2017), as well as for rhesus macaques (Silver and Watkins 2017). It is intriguing to observe that in these different primate species, the ability to control disease may be linked to specificities that share features with HLA-B27 or -B57 allotypes. In their turn, fish and amphibians, such as frogs, have turned out to represent valuable model systems, helping us to understand how some representatives of these species are dealing with challenges presented by infectious disease (Wilson 2017) (Fu and Waldman 2017).

One of the main tasks of NK cells is to scan for the absence of MHC class I at the cell surface. It is known that pathogens - but also the MHC expression pathways in cancer - may be manipulated. One of the contributions in this special issue is devoted to transmissible tumours, which are ravaging the contemporary populations of Tasmanian Devils (Caldwell and Siddle 2017). By the same token, a detailed understanding of the biological role of KIR genes may help us to battle cancer and to develop tailor-made therapies (Malmberg et al. 2017). The recognition of 'self and non-self' plays an important role in the first stages of life. The genetic make-up of the parents, and in particular of the mother and child, plays an important role in the biology of pregnancy (Colucci 2017). In a similar context, whether KIR receptors in 
humans recognise self or altered-self HLA-C structures is reviewed by another research team (Hilton and Parham 2017). We can also read that not only the classical (HLA-A, -B, and -C) but also the non-classical MHC gene products such as HLA-E, -F, and -G genes may play a significant role in reproductive biology (Persson et al. 2017). Several MHC genes are associated with a high risk of developing autoimmune diseases or a food intolerance. One contribution highlights the impact of processed MHC epitopes on the severity of arthritis (Kampstra and Toes 2017), whereas another paper highlights the impact of host genetics and interaction with food components in concert with post-translational modifications (Sollid 2017). Another intriguing phenomenon has emerged from studies involving the side effects of particular types of medicine, which may cause problems for patients with the 'wrong' type of host genetics. The role of HLA genes in pharmacogenomics is highlighted by an Australian team (McCluskey et al. 2017). Last but not least, the discovery of the MHC was propelled by transplantation research. It is fascinating to see that we are now beginning to understand that allo-HLA reactivity during a transplantation may be triggered by infectious pathogens that are shaping our immune repertoire (D’Orsogna et al. 2017).

The goal of this issue is to provide a comprehensive overview of the current state of the art relating to MHC/KIR genes in health and disease. On behalf of my co-editors, I would like to thank all contributors for their tremendous input. We also sincerely hope that our readers will enjoy this special issue of Immunogenetics.

\section{References}

Caldwell A \& Siddle HV (2017) The role of MHC genes in contagious cancer: the story of Tasmanian Devils. Immunogenetics 69: In press

Carrington M \& Naranbhai V (2017) Host genetic variation and HIV disease: from mapping to mechanism. Immunogenetics 69: In press

Colucci F (2017) The role of KIR and HLA interactions in pregnancy complications. Immmunogenetics 69: In press.

De Groot NG, Heijmans C, Bontrop RE (2017) AIDS in chimpanzees; the role of MHC genes. Immunogenetics 69: In press

D’Orsogna LL, van den Heuvel H, van Kooten C, Heidt S, Claas FJH (2017) Infectious pathogens may trigger specific allo-HLA reactivity via multiple mechanisms. Immunogenetics 69: In press

Fu M \& Waldman B (2017) Major histocompatibility complex variation and the evolution of resistance to amphibian chytridiomycosis. Immunogenetics: In press

Hilton HG \& Parham P (2017) Missing or altered self: human NK cell receptors that recognize HLA-C. Immunogenetics 69: In press

Kampstra A, Toes R (2017) HLA class II and rheumatoid arthritis: the bumpy road of revelation. Immunogenetics 69: In press.

Kelley N \& Trowsdale J (2017) Introduction: MHC/KIR and governance of specificity Immunogenetics 69: In press.

Malmberg KJ, Sohlberg E, Goodridge JP, Ljunggren H-G (2017) Immune selection during tumor checkpoint inhibition therapy paves way for NK-cell "missing self" recognition. Immunogenetics 69: In press

McCluskey J, Illing PT, Purcell AW (2017) The role of HLA genes in pharmacogenomics: unravelling HLA associated adverse drug reactions. Immunogenetics 69: In press.

Persson G, Nascimento W, Nilsson LL, Hviid TV (2017) HLA-Class Ib in pregnancy and pregnancy-related disorders Immunogenetics 69: In press.

Silver ZA \& Watkins DI (2017) The role of MHC class I gene products in SIV infection in macaques. Immunogenetics 69: In press

Sollid L (2017) The role of MHC class II genes and post-translational modifications in celiac disease. Immunogenetics 69: In press

Wilson AB (2017) MHC and adaptive immunity in fish. Immunogenetics 69: In press 